\title{
Can the height of the parotid tumor be a predictor of malignancy?
}

\author{
${\text { Gene } \mathrm{Huh}^{1} \wedge}^{\wedge}$, Jae-Cheul $\mathrm{Ahn}^{2} \wedge$, Wonjae Cha ${ }^{1} \wedge$, Woo-Jin Jeong ${ }^{1 \wedge}$ \\ ${ }^{1}$ Department of Otorhinolaryngology-Head \& Neck Surgery, Seoul National University Bundang Hospital, Seoul National University College \\ of Medicine, Seongnam, Korea; ${ }^{2}$ Department of Otorhinolaryngology-Head and Neck Surgery, CHA Bundang Medical Center, CHA University, \\ Seongnam, Korea \\ Contributions: (I) Conception and design: WJ Jeong; (II) Administrative support: WJ Jeong; (III) Provision of study materials or patients: WJ Jeong, \\ W Cha; (IV) Collection and assembly of data: G Huh, JC Ahn; (V) Data analysis and interpretation: G Huh, JC Ahn, W Cha; (VI) Manuscript \\ writing: All authors; (VII) Final approval of manuscript: All authors. \\ Correspondence to: Woo-Jin Jeong, MD, PhD. Department of Otorhinolaryngology-Head \& Neck Surgery, Seoul National University College \\ of Medicine, Seoul National University Bundang Hospital, 82, Gumi-ro 173 Beon-gil, Bundang-gu, Seongnam-si, Gyeonggi-do 13620, Korea. \\ Email: safar@snubh.org.
}

Background Accurate diagnosis of malignancy in the parotid gland before surgery is often challenging.
Various clues should be used to increase the index of suspicion for malignancy. We hypothesized that
malignant lesions of the parotid gland are located at the superior part of the gland compared to benign ones.
Methods: A total of 169 consecutive patients were included in this study whose medical records were
retrospectively reviewed. Benign and malignant tumors were compared in size, height difference from five
anatomical landmarks: hard palate, mastoid tip, earlobe, condylar head, and mandibular notch. The cutoff
heights from significant landmarks (hard palate, condylar head) were estimated with ROC analysis and chi-
square test.

Results: Twenty-nine patients (17.2\%) were diagnosed with malignant and 140 patients (82.8\%) as benign. The height differed significantly between benign and malignant tumors when the reference point was set for the hard palate $(\mathrm{P}=0.024)$ and the condylar head $(\mathrm{P}=0.049)$, with the cutoff height from reference points to be $22.5,51.5 \mathrm{~mm}$, respectively. Diagnostic values of parotid level difference presented higher sensitivity $(75.9 \%$ for hard palate, $72.4 \%$ for condylar head vs. $47.8 \%$ for fine needle aspiration cytology) and lower false negative rate $(24.1 \%$ for hard palate, $27.6 \%$ for condylar head $v$ s. $52.2 \%$ for fine needle aspiration cytology) compared to fine needle aspiration cytology.

Conclusions: Malignant tumors of the parotid gland tend to locate at the superior part of the gland compared to benign tumors. Parotid tumors lying cephalad should raise an index of suspicion for malignancy. Height of the tumor in the parotid gland should be deliberately considered during the first encounter of the patient, which in turn could curate the next step in the diagnostic approach and treatment planning.

Keywords: Parotid neoplasm; physical examination; routine diagnostic test

Submitted Oct 02, 2020. Accepted for publication Dec 13, 2020.

doi: $10.21037 /$ gs-20-741

View this article at: http://dx.doi.org/10.21037/gs-20-741

^ORCID: Gene Huh, 0000-0003-4487-1361; Jae-Cheul Ahn, 0000-0002-1191-4209; Wonjae Cha, 0000-0001-7292-9474; Woo-Jin Jeong, 00000001-8577-6964. 


\section{Introduction}

Salivary gland tumors are known to be rare, which account for $6 \%$ of head and neck tumors, and parotid tumors comprise $80 \%$ of all salivary tumors $(1,2)$. Although most of the salivary gland tumor requires surgical resection, the planning of treatment should be made with careful assessment of the tumor, since the broader extent of surgical intervention implies higher risk of morbidities, including facial nerve injury (3). Distinguishing the tumor type preoperatively, whether benign or malignant, and accurate preoperative planning is imperative in the successful outcome of parotid surgery (4). Hence, various diagnostic methods are used alone or in combination. Computed tomography (CT) and magnetic resonance imaging (MRI) are commonly used to evaluate the tumor size, location, and involvement of neck lymph nodes or other adjacent structures. Ultrasonography (US) with fine-needle aspiration cytology (FNAC) and/or core needle biopsy $(\mathrm{CNB})$ is considered first when distinguishing the benign lesion from the malignant one (5).

Although FNAC/CNB might not provide a definitive diagnosis of the tumor, together with histopathologic study, it is considered as a gold standard for preoperative diagnostic evaluation (6). However, the accuracy of preoperative diagnosis with FNAC/CNB is often challenging for pathologists, and malignant lesions are occasionally misinterpreted as benign lesions. Schmidt et al. (7), provided a meta-analysis on the diagnostic accuracy of FNAC, presenting the average sensitivity of $80 \%$ and specificity of $97 \%$. Song et al. (8) reported that sensitivity and specificity of FNAC were demonstrated to be $58.2 \%$ and $98.6 \%$, respectively, when distinguishing the benign lesion from the malignant lesion. Therefore, multiple diagnostic methods should all be taken into consideration when approving the diagnosis of parotid tumors, and in turn, arranging the surgical plan.

One of the first and most fundamental diagnostic approaches in clinical practice is physical examination. Although advanced technological diagnostic tools may provide detailed information of the disease, physical examination is essential and must be considered first in the diagnostic step. However, studies regarding the physical presentation of parotid gland tumors and its application in the diagnostic approach are lacking.

The objective of this study was to identify the physical presentation of parotid tumors which could provide a primary clue distinguishing the malignant lesion from the benign. From our clinical experience, we hypothesized that malignant lesions of the parotid gland tend to locate at the superior part of the gland compared to benign lesions, which tends to locate at the inferior part of the gland (Figure 1).

We present the following article in accordance with the STARD reporting checklist (available at http://dx.doi. org/10.21037/gs-20-741).

\section{Methods}

Clinical data of 191 consecutive patients that had undergone parotidectomy between August 2003 and July 2011 in a tertiary referral center, catering to both referral patients and patients from the community, were extracted from the data warehouse of our institute. A retrospective review of electronic medical records was conducted for each patient after obtaining approval from the Institutional Review Board (B-2005/613-104). The study was conducted in accordance with the Declaration of Helsinki (as revised in 2013). Individual consent for this retrospective analysis was waived. Patients were evaluated for their age at operation, sex, side of the tumor, radiologic data, and pathologic data. Patients that had undergone parotidectomy for the primary lesion within the parotid gland were included. Patients lacking radiologic or clinical data, and those with lesions not originating from the parotid gland or duct tissues were excluded. Since the intention of this study involves parotid gland origin tumors, nineteen patients with the final pathologic diagnosis of schwannoma, lipoma, branchial cleft cyst, lymphoepithelial cyst and lymphoma were excluded. Three patients were excluded due to the lack of appropriate diagnostic workup records. Overall, 169 patients were eligible for this study. None of the patients included presented obvious clinical signs of malignancy such as facial nerve paralysis, significantly palpable neck nodes, or skin involvement of tumor.

Radiologic results of CT or MRI scan was evaluated to locate the parotid tumor precisely. The size of the tumor was estimated by the largest diameter based on at least three consecutive sections on CT or MRI images. Level (height) of the tumor was determined by the following method. The axial cut that contains the epicenter of the tumor was indicated as the reference level of the tumor. Then the distance of the tumor level (height) was compared with five other anatomical landmarks. Five anatomical landmarks were selected for this study: superior surface of hard palate $(\mathrm{H})$, inferior end of mastoid tip $(\mathrm{M})$, inferior end of earlobe (E), center of condylar head (C), and mandibular notch (N). These five landmarks were selected because they were 

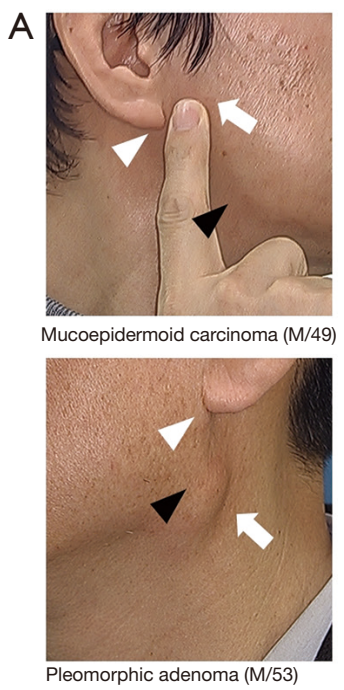
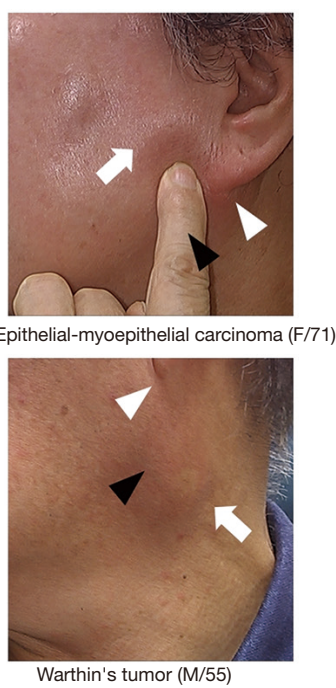

B

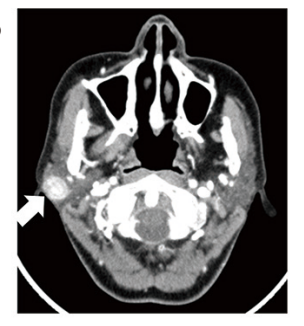

Mucoepidermoid carcinoma (F/59)

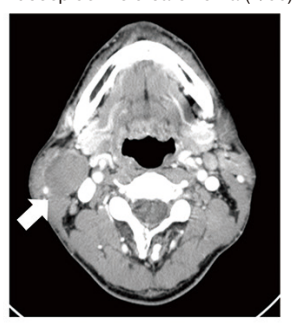

Pleomorphic adenoma (M/42)

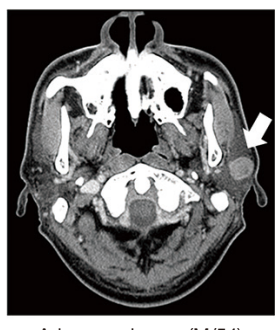

Adenocarcinoma (M/54)

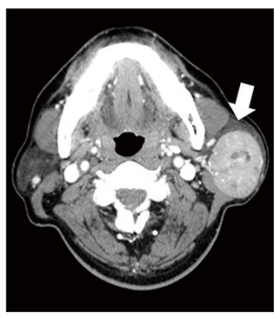

Warthin's tumor (M/60)

Figure 1 Clinical presentation of parotid tumor. (A) Physical presentation of malignant parotid tumor (upper column) located at relatively higher level and benign tumors (lower column) at lower level of parotid gland. Tumor (white arrow), ear lobule (white arrow tip), and mandible angle (black arrow tip) are marked. (B) Typical computed tomography of malignant parotid tumors (upper column) located at relatively higher level and benign parotid tumors (lower column) located at lower level of parotid gland. Tumors are marked with a white arrow.

clearly distinguishable on CT or MRI and all lie adjacent to the parotid gland with relatively constant location compared to one another (Figure S1). Using this method, five values of tumor level measured from each landmark were obtained for all patients. The tumor level was measured from each landmark by numbering the axial section in between. The mean cut size of CT was $3.11 \mathrm{~mm}$ and it was $4.75 \mathrm{~mm}$ for MRI. Out of 163 patients with CT, 139 were taken under $3 \mathrm{~mm}$ cut size, and out of 13 patients with MRI, 7 patients were taken under $5 \mathrm{~mm}$ cut size. The level difference was calculated by multiplying the cut size and the cut number in between for each modality. The diagnosis of the parotid tumor was confirmed by postoperative pathology. The size and height difference from the reference points were measured for the diagnostic group of pleomorphic adenomas, Warthin's tumors, other benign tumors, mucoepidermoid carcinomas, and other malignant tumors. The average distance of the tumor center from each reference point was measured and compared among benign and malignant tumors. The diagnostic values of setting the diagnostic standards as the cutoff point from the significant reference points were compared to the diagnostic value of FNAC performed in this population. Sensitivity, specificity, accuracy, positive predictive value, negative predictive value, prevalence, false positive rate, and false negative rate were achieved from each diagnostic method for comparison.

\section{Statistical analysis}

For statistical analysis of this study, the level difference from each anatomical landmark for each diagnosis of the tumor were compared with one-way analysis of variance (ANOVA). Data were presented as means \pm standard deviations (SD). The tumor level (height) difference from each anatomical landmark was compared between malignant and benign tumor groups by Student's $t$-test. ROC curves were analyzed of the level difference from significant reference points to determine the best cut-off value for distinguishing the malignant tumor from benign ones. The area under the curve (AUC), 95\% confidence interval, and significance value were calculated, offering the level of the tumor characteristic of the ROC curve. Sensitivity and specificity were obtained for each value of the scale and to determine the optimal cutoff, then the statistical significance was calculated with chi-square test. The Student's $t$-test and one-way ANOVA were conducted using IBM Statistical Package for the Social Sciences version 20.0 (SPSS Inc., Chicago, IL, USA). ROC curve analysis and chi-square test were performed with Graphpad Prism 5.0 (GraphPad Software, San Diego, CA, USA). 
Table 1 Comparison of parotid tumor level from each reference point $(\mathrm{n}=169)$

\begin{tabular}{lccc}
\hline Variable & Benign $(\mathrm{n}=140)$ & Malignant $(\mathrm{n}=29)$ & $\mathrm{P}$ (unpaired $t$-test) \\
\hline Size $(\mathrm{mm})$ & $24.5 \pm 9.1$ & $27.3 \pm 13.2$ & 0.169 \\
Hard palate & $28.0 \pm 23.9$ & $16.8 \pm 24.5$ & $0.024^{*}$ \\
Mastoid tip & $19.8 \pm 18.8$ & $13.5 \pm 20.8$ & 0.106 \\
Ear lobe tip & $14.3 \pm 18.9$ & $6.9 \pm 21.9$ & 0.064 \\
Condylar head & $54.1 \pm 25.3$ & $43.9 \pm 24.7$ & $0.049^{*}$ \\
Mandibular notch & $36.2 \pm 23.3$ & $29.7 \pm 23.5$ & 0.137 \\
\hline
\end{tabular}

*, statistically significant.

A

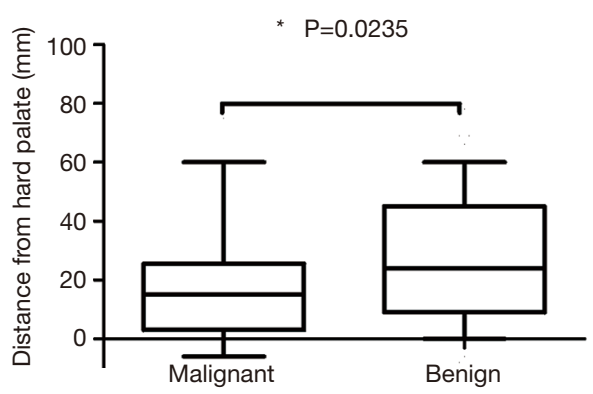

B

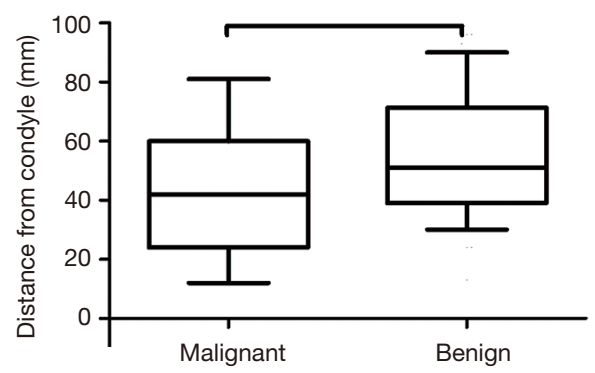

Figure 2 Comparison of the level measured from reference point between malignant and benign parotid tumor. (A) Distance from the superior level of hard palate differed significantly among malignant and benign parotid tumors (unpaired $t$-test, $\mathrm{P}=0.0235$ ). (B) Distance from condylar head differed significantly among malignant and benign parotid tumors (unpaired $t$-test, $\mathrm{P}=0.0488)$. ${ }^{*}, \mathrm{P}<0.05$.

\section{Results}

Among the 169 patients, the median age was 49 years (22-83 years old), and 82 patients were male $(48.5 \%)$ and 87 were female $(51.5 \%) .96$ tumors $(56.8 \%)$ were located on the left side and $73(43.2 \%)$ on the right side. Malignant tumors ( $\mathrm{n}=29)$ included mucoepidermoid carcinomas $(\mathrm{n}=16)$, epithelial-myoepithelial carcinomas $(n=4)$, adenoid cystic carcinomas $(n=3)$, acinic cell carcinomas $(n=3)$, etc. (lymph adenocarcinoma, carcinoma ex pleomorphic adenoma, salivary duct carcinoma). Benign tumors $(\mathrm{n}=140)$ consisted of pleomorphic adenomas $(n=96)$, Warthin's tumor $(n=35)$, basal cell adenomas ( $n=7)$, etc. (fibromatosis, cystadenoma). There were 163 patients evaluated with CT (136 benign and 27 malignant tumors), 13 with MRI (9 benign and 4 malignant tumors). Seven patients were evaluated with both CT and MRI ( 5 benign and 2 malignant tumors). Average size of the tumor was $27.3 \mathrm{~mm}$ for malignant and $24.5 \mathrm{~mm}$ for benign tumors.

Each diagnostic group represented a statistically significant difference in tumor levels from the five reference points (H, M, E, C, N) (Table S1). The average distance from hard palate $(\mathrm{H})$, mastoid $(\mathrm{M})$, ear lobe $(\mathrm{E})$, condylar head $(\mathrm{C})$, and mandibular notch $(\mathrm{N})$ were measured to be 28.0, 19.8, 14.3, 54.1, and $36.2 \mathrm{~mm}$, respectively for benign tumors, and 16.8, 13.5, 6.9, 43.9, and $29.7 \mathrm{~mm}$ for malignant tumors, respectively (Table 1). The distance (height) differed significantly between benign and malignant tumors when the reference point was set for hard palate $(\mathrm{H}, \mathrm{P}=0.024)$ and condylar head (C, $\mathrm{P}=0.049)$, as displayed in Figure 2.

To set the cutoff level (height) to distinguish benign and malignant tumors, ROC curve analysis was conducted for the two significant points of the hard palate $(\mathrm{H})$ and condylar head (C) (Figure S2). The AUC was estimated to be $0.633(\mathrm{H})$ and $0.625(\mathrm{C})$, which were statistically significant $(\mathrm{P}=0.025$ and 0.034 , respectively). The cutoff height for hard palate $(\mathrm{H})$ was the most optimal at $22.5 \mathrm{~mm}$ (chi-square test, $\mathrm{P}=0.007$ ), with a sensitivity of $75.9 \%$ and a specificity of $51.4 \%$. Likewise, the cutoff height for condylar head (C) was the most optimal at $51.5 \mathrm{~mm}$ (chi-square test, $\mathrm{P}=0.039$ ), with a sensitivity of $72.4 \%$ and a specificity of $48.6 \%$ (Table 2). 
Table 2 Clinical presentation with cutoff value applied from hard palate $\left(\mathrm{P}=0.007^{*}\right)$ and condylar head $\left(\mathrm{P}=0.039^{*}\right)$

\begin{tabular}{lccc}
\hline Variable & Malignant & Benign & Total \\
\hline Total & 29 & 140 & 169 \\
Level from hard palate & & & \\
$<22.5 \mathrm{~mm}$ & 22 & 68 & 90 \\
$>22.5 \mathrm{~mm}$ & 7 & 72 & 79 \\
Level from condylar head & & & \\
$<51.5 \mathrm{~mm}$ & 21 & 72 & 93 \\
$>51.5 \mathrm{~mm}$ & 8 & 68 & 76 \\
\hline
\end{tabular}

*, statistical significance estimated with chi-squared test.

Table 3 Diagnostic values of FNAC, parotid tumor level difference from hard palate and condylar head

\begin{tabular}{lccc}
\hline Variable & FNAC & Hard palate & Condylar head \\
\hline Sensitivity (\%) & 47.8 & 75.9 & 72.4 \\
Specificity (\%) & 98.3 & 51.4 & 48.6 \\
Accuracy (\%) & 90.1 & 55.6 & 52.7 \\
PPV (\%) & 84.6 & 24.4 & 22.6 \\
NPV (\%) & 90.7 & 91.1 & 89.5 \\
Prevalence (\%) & 16.2 & 17.2 & 17.2 \\
FPR & 1.7 & 48.6 & 51.4 \\
FNR & 52.2 & 24.1 & 27.6 \\
\hline
\end{tabular}

FNAC, fine needle aspiration cytology; PPV, positive predictive value; NPV, negative predictive value; FPR, false positive rate; FNR, false negative rate.

The diagnostic values are compared among FNAC, and diagnosis by the reference point of superior surface of hard palate and condylar head (Table 3). Although FNAC demonstrated better results for specificity, accuracy, and positive predictive, diagnosis by parotid level presented a higher sensitivity $(75.9 \%$ for $\mathrm{H}, 72.4 \%$ for $\mathrm{C} v s .47 .8 \%$ for FNAC) and with a lower false negative rate $(24.1 \%$ for $\mathrm{H}$, $27.6 \%$ for $\mathrm{C} v$ s. $52.2 \%$ for FNAC), accordingly.

\section{Discussion}

Our study demonstrates for the first time that malignant parotid tumors tend to locate at a higher level (cephalad) in the parotid gland compared to benign tumors. Moreover, our study also determined the anatomical landmarks that could be used as a guide in distinguishing malignant tumors from benign tumors. Due to the low diagnostic accuracy of FNAC for parotid malignancy, physical examination per se can augment the index of suspicion for malignancy in a way even better than FNAC itself. This result can change the first impression of the parotid tumor during physical examination and consequently aid in guiding further diagnostic work-up.

Preoperative diagnosis of parotid gland tumors is often indefinite, and it requires several diagnostic tools to obtain clear evidence. Since the diagnosis of benign and malignant tumors could lead to a completely different treatment plan, there is an ongoing debate of the optimal diagnostic methods on determining whether the tumor is benign or malignant. Lee et al. recently reported the preoperative diagnostic accuracy of benign and malignant parotid gland tumors to be $100 \%$ and $57 \%$, respectively (9), which implies that diagnostic accuracy of malignant tumors is relatively low, and many cases are diagnosed postoperatively. Likewise, various studies have attempted to point out the characteristic features of parotid tumors to improve the accuracy of diagnosis, but most of the reports mainly focused on diagnosis using imaging tools, determining the involvement of adjacent structures (e.g., facial nerve, etc.) and verifying the tumor location by superficial or deep lobe in which it is contained (10-12).

The present study is the first to discuss the issue of distinguishing the diagnosis of parotid gland tumors based on physical examination. Generally, physical examination of the parotid gland tumor is directed to determine the presence of facial nerve palsy and the depth of tumor location. Since current physical examination holds low reliability for a definite diagnosis, the purpose of physical examination lies in clarifying the surgical approach and extent of excision of parotidectomy. Under this circumstance, the diagnostic approach for the parotid gland tumor is fully dependent on imaging and pathologic workups. Although it is commonly accepted that imaging and pathologic workup provides more accurate and practical information compared to physical presentation, physical examination still should be performed carefully if it could present additional clues for diagnosis. For this intention, bone structures were favored as a landmark in this study over other options, such as facial nerve trunk. Although it may not be suitable enough to set the diagnostic standards of distinguishing parotid malignancy, bone landmarks are applicable as an objective indicator on surface anatomy, 
providing the advantage of physical examination as an additional diagnostic clue. Facial nerve trunk is a conventional landmark of parotid tumor, however facial nerve trunk is hard to visualize on surface anatomy without advanced MRI workups. It is well known that MRI provides more diagnostic values in diagnosis of parotid tumors compared to CT, concerning soft tissue differentiation (13). However, CT can also provide appropriate information in tumor characterization and their relationship to adjacent structures (14). Moreover, the cost of CT is remarkably low compared to MRI due to the insurance policy. Thus, considering the cost-effectiveness in actual practice, majority of the population in our study conducted CT as radiologic diagnosis method.

The main finding of this study is that tumor location in the parotid gland can provide information that can distinguish malignant from benign tumors. If the tumor is located at the superior portion of the parotid gland, the degree of alertness for the possibility of malignancy should be maintained, even if benign is more likely on other studies. This was especially notable considering that using the tumor presentation level performed with better sensitivity and false negative rate compared to FNAC (Table 3). The diagnostic value of FNAC in this study was consistent with previous reports (6-8), which presented with higher specificity and accuracy compared to those using the parotid tumor level difference from the superior surface of the hard palate and the condylar head. Although correctly pointing out the benign tumor could improve the efficiency of clinical flow, it is more critical for the patient not to miss on the diagnosis of malignant tumors. Thus, even if diagnostic values of using the parotid tumor level are not valid nor reliable enough to be accepted as a definitive diagnostic process, a diagnostic attempt based on the parotid tumor level could convey substantial information on distinguishing malignant from benign tumors. Moreover, due to its low false negative rate, it may be a valuable consideration at the first step of diagnosis.

According to our study, the cutoff heights from each reference points were most significant at $22.5 \mathrm{~mm}$ inferior from the superior surface of the hard palate, and $51.5 \mathrm{~mm}$ inferior from the condylar head (Table 2). These values point to the level roughly above the mandibular angle and the lower portion of the mandible ramus, evaluated from the surface anatomy (15). It may be difficult to apply the accurate cutoff height just by using the surface anatomy, without referring to CT or MRI. Nonetheless, inspection of the parotid tumor level regarding the height difference from anatomical landmark is suggested considering the result of this study. The findings of this study may not provide a conclusive diagnosis. However, it offers considerable information in the diagnostic process, especially concerning the relevant value of its false negative rate. Therefore, evaluation of parotid tumor by its location among the parotid gland should be considered to indicate possible malignant lesion, which may lead to confirmation studies such as core-needle biopsy or intra-operative frozen biopsy, or even suggesting an en bloc resection as a surgical plan.

There are several limitations to this study. First, the retrospective design of this study has its own limitation. Three of the patients were excluded due to insufficient data retrieved, which might have affected the fidelity of the study. This study involves rather small number of population and malignant tumors concerning the study period of 8 years, which is during the early history of the center. The utmost population was involved but there were clear limitations in maximizing the study population. Moreover, relying on medical record data might raise some questions of actual symptoms and findings of the patient, which may have influenced the impression of disease. A crucial weakness of this study is that Warthin's tumor could have largely influenced the significance in the lower level presentation of benign tumors. It is well known that Warthin's tumor is often located at the tail of the parotid gland. In this study, Warthin's tumor comprises $25 \%$ of all benign tumors, presenting with significantly low level (Table S1). However, concerning that Warthin's tumor are the second most common benign parotid tumor (16), it is assumed appropriate in this study to classify the benign tumor group including the Warthin's tumor. Another weakness is that even though the anatomical landmarks presented in this study are somewhat analogous to each other, only 2 reference points $(H, C)$ out of 5 candidates $(H$, $\mathrm{M}, \mathrm{E}, \mathrm{C}, \mathrm{N}$ ) presented with significant results, which is not clearly understood. The variability of maxilla and mandible height is known to be affected by sex, age, and dental state of the patient $(17,18)$, but coarse measures regarding the anatomical landmarks in this study $(\mathrm{H}, \mathrm{M}, \mathrm{E}, \mathrm{C}, \mathrm{N})$ are not previously reported. Since the patient factor of age, sex, and dental state were not considered in the analysis, it is only suspected that various size of the mandible, maxilla, or the external ear could have influenced the result. Moreover, the degree of head extension/flexion of each patient during imaging workup may have negatively affected the 
consistency of the measurement, therefore a well-designed prospective study minimizing the confounding factor is necessary for future studies.

\section{Conclusions}

Malignant parotid gland tumors are relatively rare and preoperative diagnosis is often challenging even with various diagnostic methods. Since the treatment mostly requires surgical intervention which could impair the patient's facial nerve function, the diagnosis of parotid tumor, whether benign or malignant, should be considered carefully. The result of this study demonstrates that malignant tumor of parotid gland tends to locate in the superior part (cephalad) of the gland, whereas the benign tumor tends to locate in the inferior part (caudad) of the parotid gland. Although diagnostic values are not valid for an authoritative diagnostic workup, examining the parotid tumor level during the first step of diagnosis could raise the index of suspicion for malignancy, which could eventually lead to arranging a curated diagnostic workup.

\section{Acknowledgments}

Funding: We are grateful for the support provided for this study by the SNUBH Research Fund (Grant Number: 032012-019).

\section{Footnote}

Reporting Checklist: The authors have completed the STARD reporting checklist. Available at http://dx.doi.org/10.21037/ gs-20-741

Data Sharing Statement: Available at http://dx.doi. org/10.21037/gs-20-741

Conflicts of Interest: All authors have completed the ICMJE uniform disclosure form (available at http://dx.doi. org/10.21037/gs-20-741). The authors have no conflicts of interest to declare.

Ethical Statement: The authors are accountable for all aspects of the work in ensuring that questions related to the accuracy or integrity of any part of the work are appropriately investigated and resolved. The study was conducted in accordance with the Declaration of Helsinki (as revised in 2013). The study was approved by the institutional review boards of Seoul National University Bundang Hospital (approvement number: B-2005/613-104) and individual consent for this retrospective analysis was waived.

Open Access Statement: This is an Open Access article distributed in accordance with the Creative Commons Attribution-NonCommercial-NoDerivs 4.0 International License (CC BY-NC-ND 4.0), which permits the noncommercial replication and distribution of the article with the strict proviso that no changes or edits are made and the original work is properly cited (including links to both the formal publication through the relevant DOI and the license). See: https://creativecommons.org/licenses/by-nc-nd/4.0/.

\section{References}

1. Stenner M, Klussmann JP. Current update on established and novel biomarkers in salivary gland carcinoma pathology and the molecular pathways involved. Eur Arch Otorhinolaryngol 2009;266:333-41.

2. Sowa P, Goroszkiewicz K, Szydelko J, et al. A review of selected factors of salivary gland tumour formation and malignant transformation. BioMed Res Int 2018;2018:2897827.

3. Bussu F, Rigante M, Giglia V, et al. Clinical history, prognostic factors, and management of facial nerve in malignant tumors of the parotid gland. Clin Exp Otorhinolaryngol 2014;7:126.

4. Lombardi D, McGurk M, Vander Poorten V, et al. Surgical treatment of salivary malignant tumors. Oral Oncol 2017;65:102-13.

5. Romano EB, Wagner JM, Alleman AM, et al. Fine-needle aspiration with selective use of core needle biopsy of major salivary gland tumors. Laryngoscope 2017;127:2522-7.

6. Sungur N, Akan IM, Ulusoy MG, et al. Clinicopathological evaluation of parotid gland tumors: a retrospective study. J Craniofac Surg 2002;13:26-30.

7. Schmidt RL, Hall BJ, Layfield LJ. A systematic review and meta-analysis of the diagnostic accuracy of ultrasoundguided core needle biopsy for salivary gland lesions. Am J Clin Pathol 2011;136:516-26.

8. Song IH, Song JS, Sung CO, et al. Accuracy of core needle biopsy versus fine needle aspiration cytology for diagnosing salivary gland tumors. J Pathol Transl Med 2015;49:136.

9. Lee WH, Tseng TM, Hsu HT, et al. Salivary gland tumors: A 20-year review of clinical diagnostic accuracy at 
a single center. Oncol Lett 2014;7:583-7.

10. Christensen RK, Bjorndal K, Godballe C, et al. Value of fine-needle aspiration biopsy of salivary gland lesions. Head Neck 2010;32:104-8.

11. Taylor MJ, Serpell JW, Thomson P. Preoperative fine needle cytology and imaging facilitates the management of submandibular salivary gland lesions. ANZ J Surg 2011;81:70-4.

12. Sagiv D, Witt RL, Glikson E, et al. Warthin tumor within the superficial lobe of the parotid gland: a suggested criterion for diagnosis. Eur Arch Otorhinolaryngol 2017;274:1993-6.

13. Koyuncu M, Şeşen T, Akan H, et al. Comparison of computed tomography and magnetic resonance imaging in the diagnosis of parotid tumors. Otolaryngol Head Neck Surg 2003;129:726-32.

14. Reginelli A, Clemente A, Renzulli M, et al. Delayed

Cite this article as: Huh G, Ahn JC, Cha W, Jeong WJ. Can the height of the parotid tumor be a predictor of malignancy? Gland Surg 2021;10(2):721-728. doi: 10.21037/gs-20-741 enhancement in differential diagnosis of salivary gland neoplasm. Gland Surg 2019;8:S130.

15. Sairam V, Geethamalika MV, Kumar PB, et al. Determination of sexual dimorphism in humans by measurements of mandible on digital panoramic radiograph. Contemp Clin Dent 2016;7:434-9.

16. Guntinas-Lichius O, Klussmann JP, Wittekindt C, et al. Parotidectomy for benign parotid disease at a university teaching hospital: outcome of 963 operations. Laryngoscope 2006;116:534-40.

17. Chandra P, Govindaraju P, Chowdhary R. Radiographic evaluation of anatomical variables in maxilla and mandible in relation to dental implant placement. Indian J Dent Res 2016;27:344-7.

18. Panchbhai AS. Quantitative estimation of vertical heights of maxillary and mandibular jawbones in elderly dentate and edentulous subjects. Spec Care Dentist 2013;33:62-9. 


\section{Supplementary}

Table S1 Size and level difference from reference points for each diagnostic group

\begin{tabular}{|c|c|c|c|c|c|c|c|c|}
\hline & Types & $\mathrm{n}$ & Size $(\mathrm{mm})$ & Hard palate & Mastoid tip & Ear lobe & Condylar head & Mandible notch \\
\hline \multirow[t]{3}{*}{ Benign } & Pleomorphic adenoma & 96 & $23.2 \pm 7.7$ & $19.3 \pm 19.0$ & $14.6 \pm 16.0$ & $9.2 \pm 16.0$ & $46.2 \pm 22.2$ & $28.9 \pm 18.8$ \\
\hline & Warthin's tumor & 35 & $29.0 \pm 11.3$ & $52.6 \pm 21.0$ & $35.5 \pm 18.1$ & $29.9 \pm 18.5$ & $75.8 \pm 22.2$ & $56.9 \pm 24.5$ \\
\hline & Other benign & 9 & $20.7 \pm 8.3$ & $25.6 \pm 15.0$ & $14.3 \pm 16.2$ & $8.1 \pm 17.7$ & $55.1 \pm 19.6$ & $33.2 \pm 13.8$ \\
\hline Malignant & MEC & 16 & $26.3 \pm 12.3$ & $19.3 \pm 24.0$ & $19.0 \pm 21.5$ & $12.4 \pm 23.1$ & $45.9 \pm 25.5$ & $31.8 \pm 22.1$ \\
\hline$P$ & One-way ANOVA & & 0.013 & $<0.001$ & $<0.001$ & $<0.001$ & $<0.001$ & $<0.001$ \\
\hline
\end{tabular}

MEC, mucoepidermoid carcinoma.
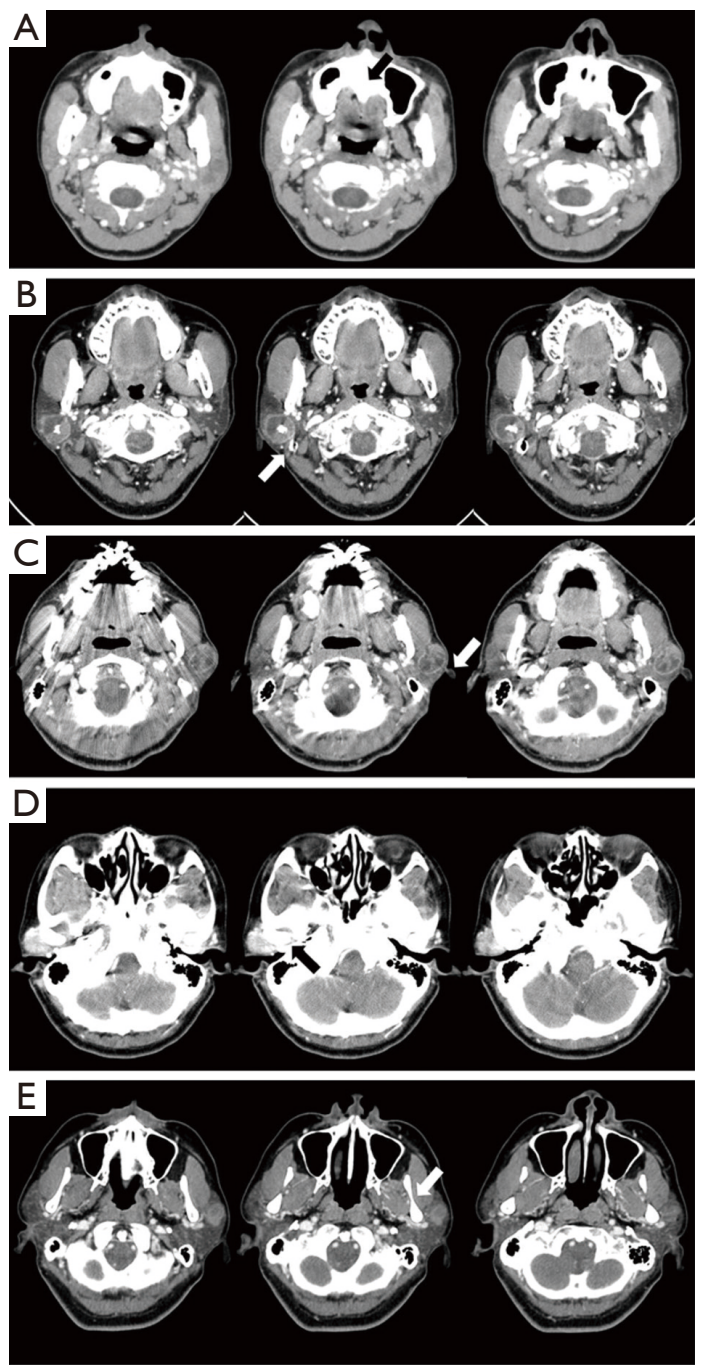

Figure S1 Consecutive images of five reference points on the computed tomography (CT) scan. (A) Superior surface of hard palate (black arrow). (B) Inferior end of mastoid tip (white arrow). (C) Inferior end of earlobe (white arrow). (D) Center of condylar head (black arrow). (E) Mandibular notch (white arrow). 

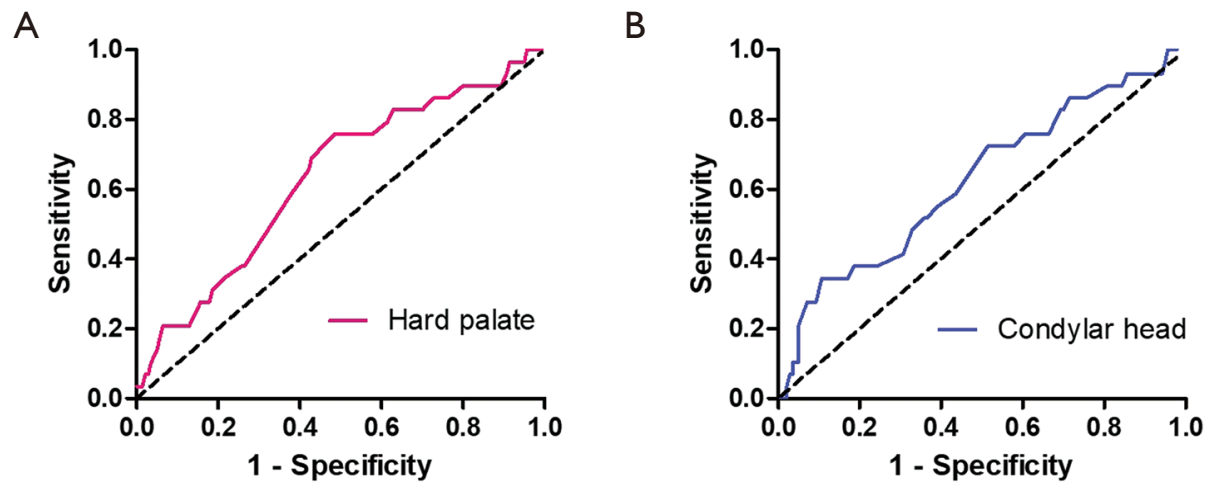

Figure S2 ROC curve analysis from two significant reference points. (A) Superior surface of hard palate (AUC =0.633, $\mathrm{P}=0.025)$. (B) Center of condylar head (AUC $=0.625, \mathrm{P}=0.034)$. 